\title{
Universal back-projection algorithm for photoacoustic computed tomography
}

\author{
Minghua Xu and Lihong V. Wang* \\ Optical Imaging Laboratory, Department of Biomedical Engineering, Texas A\&M University, 3120 TAMU, \\ College Station, Texas 77843-3120, USA
}

(Received 13 July 2004; published 19 January 2005)

\begin{abstract}
We report results of a reconstruction algorithm for three-dimensional photoacoustic computed tomography. A universal back-projection formula is presented for three types of imaging geometries: planar, spherical, and cylindrical surfaces. A solid-angle weighting factor is introduced in the back-projection formula to compensate for the variations of detection views. A method for implementing this algorithm is described. Numerical simulation is used to demonstrate the performance of the algorithm.
\end{abstract}

DOI: 10.1103/PhysRevE.71.016706 PACS number(s): 95.75.Mn, 87.57.Ce, 43.35. + d, 43.60. + d

\section{INTRODUCTION}

Photoacoustic (PA) computed tomography is based on the reconstruction of an internal PA source distribution from measurements acquired by scanning small-aperture ultrasound detectors over a surface that encloses the source under study [1]. The PA source is produced inside the sample by the thermal expansion that results from a small temperature rise, which is caused by the absorption of externally applied radiation of pulsed electromagnetic (EM) waves. This technique has great potential for applications in the biomedical field because of the advantages of ultrasonic resolution in combination with EM absorption contrast. In general, different measurement geometries need different reconstruction algorithms. The three geometries commonly used are planar, cylindrical, and spherical surfaces. In the last decade, Fourier-domain reconstruction formulas with point-detector measurements for these geometries have been derived (summarized in Ref. [1]), but they may involve multiple integrations or series summations and can be inconvenient to implement. Alternatively, some approximate time-domain backprojection reconstruction algorithms have also been reported [2-5]. In these algorithms, the acoustic property of the tissue is often assumed to be homogeneous as the speed of sound in soft tissue is relatively constant at $1.5 \mathrm{~mm} / \mu \mathrm{s}$. The unique advantage of photoacoustic imaging is its ability to detect the inhomogeneous EM absorption property of tissues when the acoustic property is relatively homogeneous. Pure acoustic property differentiation should appeal to conventional ultrasound imaging.

From a physical point of view, PA tomography (PAT) represents an inverse source problem analogous to positron emission tomography (PET), except that PAT is based on diffraction "optics" due to the diffraction of ultrasonic waves and PET is based on geometric "optics" due to the straightline propagation of $\gamma$ rays. Therefore, PAT belongs to the field of diffraction tomography. Since acoustic waves do not travel along straight lines, the projections are not line inte-

\footnotetext{
*Author to whom all correspondence should be addressed. FAX: 979-845-4450. Electronic address: LWang@tamu.edu; URL: http:// oilab.tamu.edu
}

grals, which are in contrast to those in straight-ray tomography such as PET and x-ray computed tomography (CT) [6]. Nevertheless, many imaging concepts and mathematical techniques for other imaging modalities, such as ultrasonic, $\mathrm{x}$-ray, and optical tomography, can be borrowed for PAT applications.

In this paper, we present a universal back-projection (BP) formula, which offers exact reconstructions for the three common geometries and can be easily implemented in the reconstruction. For the spherical geometry, we note that a similar inversion formula was recently reported [7]; however, it can be simplified to the formula that we present here. The paper is organized as follows. In Sec. II, the universal back-projection formula will be derived. A proof of the exactness of this formula for the three common geometries will be demonstrated. In Sec. III, a method for implementing this algorithm will be described. In Sec. IV, numerical simulations will be carried out to demonstrate the performance of the algorithm. In Sec. V, a conclusion will be provided.

\section{UNIVERSAL BACK-PROJECTION FORMULA}

According to the PA generation theory, the initial photoacoustic pressure at position $\mathbf{r}$ excited by a $\delta(t)$ EM pulse equals $p_{0}(\mathbf{r})=\Gamma(\mathbf{r}) A(\mathbf{r})[8]$, where $A(\mathbf{r})$ is a spatial EM absorption function and $\Gamma(\mathbf{r})$ is the Grüneisen parameter equal to $c^{2} \beta / C_{p}$ (c the speed of sound; $\beta$ the isobaric volume expansion coefficient, and $C_{p}$ the specific heat). The acoustic wave, $p(\mathbf{r}, t)$ at position $\mathbf{r}$ and time $t$, prompted by the initial source $p_{0}(\mathbf{r})$, satisfies the wave equation $[8,9]$

$$
\left(\boldsymbol{\nabla}^{2}-\frac{1}{c^{2}} \frac{\partial^{2}}{\partial t^{2}}\right) p(\mathbf{r}, t)=-p_{0}(\mathbf{r}) \frac{d \delta(t)}{d t}
$$

In this paper, we define the Fourier transform pair on variable $\bar{t}=c t$ as $\tilde{F}(k)=\int_{-\infty}^{+\infty} F(\bar{t}) \exp (i k \bar{t}) d \bar{t}$ and $F(\bar{t})$ $=(1 / 2 \pi) \int_{-\infty}^{+\infty} \tilde{F}(k) \exp (-i k \vec{t}) d k$, where $k=\omega / c$ and $\omega$ is an angular frequency and equal to $2 \pi f$. As shown in Fig. 1, we assume that $S_{0}$ is the measurement surface. Particularly, for the planar geometry, we assume there is another planar surface $S_{0}^{\prime}$ (parallel to $S_{0}$ ) at infinity and that the combination of $S_{0}^{\prime}$ and $S_{0}$ encloses the source $p_{0}\left(\mathbf{r}^{\prime}\right)$. For convenience, we 


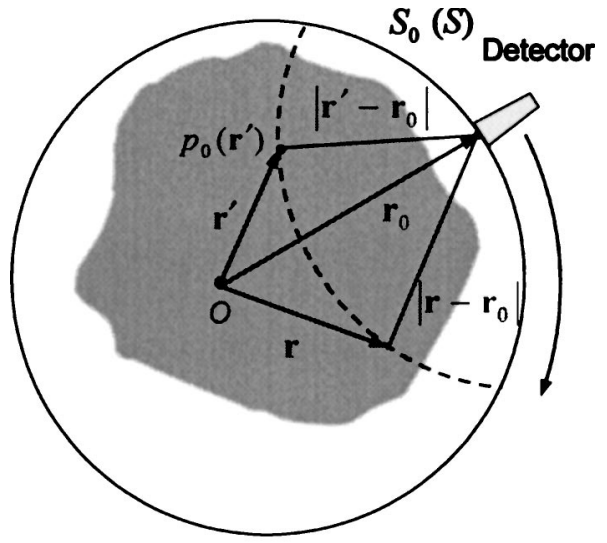

(a)

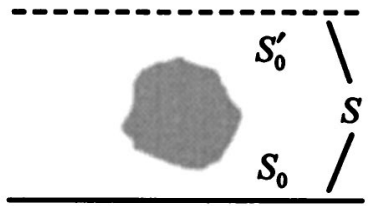

(b)

FIG. 1. (a) In the measurement, an ultrasonic detector at position $\mathbf{r}_{0}$ on a surface $S_{0}$ receives PA signals emitted from source $p_{0}\left(\mathbf{r}^{\prime}\right)$. In the reconstruction, a quantity related to the measurement at position $\mathbf{r}_{0}$ projects backward on a spherical surface with respect to position $\mathbf{r}_{0}$. (b) In the planar geometry, assume there is another surface $S_{0}^{\prime}$ at infinity and that the combination of $S_{0}$ and $S_{0}^{\prime}$ encloses the source inside.

denote $S=S_{0}+S_{0}^{\prime}$ for the planar geometry and $S=S_{0}$ for the other two geometries. Based on Green's theorem, the spectrum $\tilde{p}\left(\mathbf{r}_{0}, k\right)$ of the pressure $p\left(\mathbf{r}_{0}, \vec{t}\right)$ detected at $\mathbf{r}_{0}$ can be written in the frequency domain [10-12] as

$$
\tilde{p}\left(\mathbf{r}_{0}, k\right)=-i k \iiint_{V^{\prime}} d^{3} r^{\prime} \widetilde{G}_{k}^{\text {(out) }}\left(\mathbf{r}^{\prime}, \mathbf{r}_{0}\right) p_{0}\left(\mathbf{r}^{\prime}\right)
$$

where $V^{\prime}$ is the volume of the source $p_{0}\left(\mathbf{r}^{\prime}\right)$ and $\tilde{G}_{k}^{\text {(out) }}\left(\mathbf{r}^{\prime}, \mathbf{r}_{0}\right)$ is a Green's function: $\tilde{G}_{k}^{\text {(out) }}\left(\mathbf{r}^{\prime}, \mathbf{r}_{0}\right)$ $=\exp \left(i k\left|\mathbf{r}^{\prime}-\mathbf{r}_{0}\right|\right) /\left(4 \pi\left|\mathbf{r}^{\prime}-\mathbf{r}_{0}\right|\right)$, which corresponds to an outgoing wave.

In principle, we can construct a Dirichlet Green's function $\widetilde{G}_{k}^{(D)}\left(\mathbf{r}, \mathbf{r}_{1}\right)$ [11,12], which satisfies the boundary condition $\widetilde{G}_{k}^{(D)}\left(\mathbf{r}, \mathbf{r}_{1}\right)=0$ for $\mathbf{r}_{1}$ on $S$ and $\mathbf{r}$ inside $S$. Then, according to Green's theorem, the acoustic pressure $\widetilde{p}(\mathbf{r}, k)$ inside surface $S$ can be computed by the surface integral: $\widetilde{p}(\mathbf{r}, k)$ $=\int_{S} d S \widetilde{p}\left(\mathbf{r}_{0}, k\right)\left[\mathbf{n}_{0}^{s} \cdot \boldsymbol{\nabla}_{0} \widetilde{G}_{k}^{(D)}\left(\mathbf{r}, \mathbf{r}_{0}\right)\right]$, where $\boldsymbol{\nabla}_{0}$ denotes the gradient over variable $\mathbf{r}_{0}$ and $\mathbf{n}_{0}^{s}$ is the normal of surface $S$ pointing to the source. Since $p_{0}(\mathbf{r})=p(\mathbf{r}, \bar{t}=0)$, we get $p_{0}(\mathbf{r})$ $=1 /(2 \pi) \int_{-\infty}^{+\infty} d k \int_{S} d S \widetilde{p}\left(\mathbf{r}_{0}, k\right)\left[\mathbf{n}_{0}^{s} \cdot \nabla_{0} \widetilde{G}_{k}^{(D)}\left(\mathbf{r}, \mathbf{r}_{0}\right)\right]$. For the three common geometries, $\widetilde{G}_{k}^{(D)}\left(\mathbf{r}, \mathbf{r}_{1}\right)$ can be written in some explicit expressions [13], with which we can directly write down the reconstruction formulas that are identical to the Fourier-domain formulas summarized in Ref. [1].
Our further study shows that the Fourier-domain reconstruction formulas for the three common geometries can be further simplified into a universal back-projection formula; however, the derivations on a case-by-case basis are too extensive. For readability, we first propose the BP formula and then demonstrate the exactness of the formula. The new back-projection formula takes the following form:

$$
p_{0}^{(b)}(\mathbf{r})=\frac{1}{\pi} \int_{S} d S \int_{-\infty}^{+\infty} d k \tilde{p}\left(\mathbf{r}_{0}, k\right)\left[\mathbf{n}_{0}^{s} \cdot \nabla_{0} \widetilde{G}_{k}^{(\text {in })}\left(\mathbf{r}, \mathbf{r}_{0}\right)\right],
$$

where $\widetilde{G}_{k}^{(\mathrm{in})}\left(\mathbf{r}, \mathbf{r}_{0}\right)$ is a Green's function, $\widetilde{G}_{k}^{(\mathrm{in})}\left(\mathbf{r}, \mathbf{r}_{0}\right)$ $=\exp \left(-i k\left|\mathbf{r}-\mathbf{r}_{0}\right|\right) /\left(4 \pi\left|\mathbf{r}-\mathbf{r}_{0}\right|\right)$, which corresponds to an incoming wave. In addition, it is straightforward to rewrite Eq. (3) in the time domain (to be detailed later).

Now, we begin to prove that $p_{0}^{(b)}(\mathbf{r}) \equiv p_{0}(\mathbf{r})$ for the three common geometries. With considering Gauss's theorem [10], substituting Eq. (2) into (3) gives

$$
p_{0}^{(b)}(\mathbf{r})=\iiint_{V^{\prime}} d^{3} r^{\prime} p_{0}\left(\mathbf{r}^{\prime}\right) \mathrm{P}\left(\mathbf{r}^{\prime}, \mathbf{r}\right),
$$

where $\mathrm{P}\left(\mathbf{r}^{\prime}, \mathbf{r}\right)$ is a point-spread function (PSF), expressed by $\mathrm{P}\left(\mathbf{r}^{\prime}, \mathbf{r}\right)=\frac{1}{\pi} \int_{-\infty}^{+\infty} i k d k \int_{V_{0}} d V_{0} \boldsymbol{\nabla}_{0} \cdot\left[\widetilde{G}_{k}^{(\text {out })}\left(\mathbf{r}^{\prime}, \mathbf{r}_{0}\right) \boldsymbol{\nabla}_{0} \widetilde{G}_{k}^{(\text {in) }}\left(\mathbf{r}, \mathbf{r}_{0}\right)\right]$,

where $V_{0}$ is the volume enclosed by surface $S$.

For convenience, we denote $\boldsymbol{\nabla}^{\prime}$ and $\boldsymbol{\nabla}$ as the gradients over the variables $\mathbf{r}^{\prime}$ and $\mathbf{r}$, respectively. Since $\nabla_{0}\left|\mathbf{r}^{\prime}-\mathbf{r}_{0}\right|$ $=-\nabla^{\prime}\left|\mathbf{r}^{\prime}-\mathbf{r}_{0}\right|$ and $\nabla_{0}\left|\mathbf{r}-\mathbf{r}_{0}\right|=-\nabla\left|\mathbf{r}-\mathbf{r}_{0}\right|$, we have

$$
\begin{aligned}
\boldsymbol{\nabla}_{0} \cdot & {\left[\widetilde{G}_{k}^{\text {(out) }}\left(\mathbf{r}^{\prime}, \mathbf{r}_{0}\right) \nabla_{0} \widetilde{G}_{k}^{\text {(in) }}\left(\mathbf{r}, \mathbf{r}_{0}\right)\right] } \\
= & \left(\boldsymbol{\nabla}^{\prime} \cdot \boldsymbol{\nabla}\right) \widetilde{G}_{k}^{\text {(out) }}\left(\mathbf{r}^{\prime}, \mathbf{r}_{0}\right) \widetilde{G}_{k}^{(\text {in) }}\left(\mathbf{r}, \mathbf{r}_{0}\right) \\
& +\widetilde{G}_{k}^{\text {(out) }}\left(\mathbf{r}^{\prime}, \mathbf{r}_{0}\right) \boldsymbol{\nabla}^{2} \widetilde{G}_{k}^{\text {(in) }}\left(\mathbf{r}, \mathbf{r}_{0}\right) .
\end{aligned}
$$

The Green's functions $\widetilde{G}_{k}^{\text {(out) }}\left(\mathbf{r}^{\prime}, \mathbf{r}_{0}\right)$ and $\widetilde{G}_{k}^{\text {(in) }}\left(\mathbf{r}, \mathbf{r}_{0}\right)$ satisfy the following equations, respectively:

$$
\boldsymbol{\nabla}^{\prime 2} \widetilde{G}_{k}^{\text {(out) }}\left(\mathbf{r}^{\prime}, \mathbf{r}_{0}\right)+k^{2} \widetilde{G}_{k}^{(\text {out })}\left(\mathbf{r}^{\prime}, \mathbf{r}_{0}\right)=-\delta\left(\mathbf{r}^{\prime}-\mathbf{r}_{0}\right)
$$

and

$$
\boldsymbol{\nabla}^{2} \widetilde{G}_{k}^{(\text {in) }}\left(\mathbf{r}, \mathbf{r}_{0}\right)+k^{2} \widetilde{G}_{k}^{(\text {in) }}\left(\mathbf{r}, \mathbf{r}_{0}\right)=-\delta\left(\mathbf{r}-\mathbf{r}_{0}\right) .
$$

Multiplying Eq. (7) by $\widetilde{G}_{k}^{\text {(in) }}\left(\mathbf{r}, \mathbf{r}_{0}\right)$ and Eq. (8) by $\widetilde{G}_{k}^{\text {(out) }}\left(\mathbf{r}^{\prime}, \mathbf{r}_{0}\right)$, then subtracting them and rearranging, we obtain

$$
\begin{aligned}
\widetilde{G}_{k}^{\text {(out) }}\left(\mathbf{r}^{\prime}, \mathbf{r}_{0}\right) \boldsymbol{\nabla}^{2} \widetilde{G}_{k}^{\text {(in) }}\left(\mathbf{r}, \mathbf{r}_{0}\right) \\
=(1 / 2)\left[\left(\boldsymbol{\nabla}^{2}+\boldsymbol{\nabla}^{\prime 2}\right) \widetilde{G}_{k}^{\text {(out) }}\left(\mathbf{r}^{\prime}, \mathbf{r}_{0}\right)\right. \\
\quad \times \widetilde{G}_{k}^{\text {(in) }}\left(\mathbf{r}, \mathbf{r}_{0}\right)+\delta\left(\mathbf{r}^{\prime}-\mathbf{r}_{0}\right) \\
\left.\quad \times \widetilde{G}_{k}^{\text {(in) }}\left(\mathbf{r}, \mathbf{r}_{0}\right)-\delta\left(\mathbf{r}-\mathbf{r}_{0}\right) \widetilde{G}_{k}^{\text {(out) }}\left(\mathbf{r}^{\prime}, \mathbf{r}_{0}\right)\right] .
\end{aligned}
$$

We then substitute Eq. (9) into Eq. (6) and further substitute Eq. (6) into Eq. (5) and rewrite $\mathrm{P}\left(\mathbf{r}^{\prime}, \mathbf{r}\right)$ as a summation 
of two terms, $\mathrm{P}\left(\mathbf{r}^{\prime}, \mathbf{r}\right)=P^{(1)}\left(\mathbf{r}^{\prime}, \mathbf{r}\right)+P^{(2)}\left(\mathbf{r}^{\prime}, \mathbf{r}\right)$, where the first term is

$$
\begin{aligned}
P^{(1)}\left(\mathbf{r}^{\prime}, \mathbf{r}\right)= & \frac{1}{\pi} \int_{-\infty}^{+\infty} i k d k \int_{V_{0}} d V_{0}(1 / 2)\left[\delta\left(\mathbf{r}^{\prime}-\mathbf{r}_{0}\right) \widetilde{G}_{k}^{(\text {in })}\left(\mathbf{r}, \mathbf{r}_{0}\right)\right. \\
& \left.-\delta\left(\mathbf{r}-\mathbf{r}_{0}\right) \widetilde{G}_{k}^{\text {(out) }}\left(\mathbf{r}^{\prime}, \mathbf{r}_{0}\right)\right]
\end{aligned}
$$

and the second term is

$$
\begin{aligned}
P^{(2)}\left(\mathbf{r}^{\prime}, \mathbf{r}\right)= & \frac{1}{2 \pi}\left(\boldsymbol{\nabla}+\boldsymbol{\nabla}^{\prime}\right)^{2} \int_{-\infty}^{+\infty} i k d k \\
& \times \int_{V_{0}} d V_{0} \widetilde{G}_{k}^{\text {(out) }}\left(\mathbf{r}^{\prime}, \mathbf{r}_{0}\right) \widetilde{G}_{k}^{(\text {in })}\left(\mathbf{r}, \mathbf{r}_{0}\right) .
\end{aligned}
$$

The first term reduces to a delta function: $P^{(1)}\left(\mathbf{r}^{\prime}, \mathbf{r}\right)$ $=\delta\left(\left|\mathbf{r}^{\prime}-\mathbf{r}\right|\right) /\left(2 \pi\left|\mathbf{r}^{\prime}-\mathbf{r}\right|^{2}\right)=\delta\left(\mathbf{r}^{\prime}-\mathbf{r}\right)$. The second term involves a volume integral that depends on the measurement geometry. Particularly, when $\mathbf{r}^{\prime}=\mathbf{r}, P^{(2)}\left(\mathbf{r}^{\prime}, \mathbf{r}\right)=0$. The second term can also be rewritten as

$$
P^{(2)}\left(\mathbf{r}^{\prime}, \mathbf{r}\right)=\frac{1}{2 \pi}\left(\boldsymbol{\nabla}+\nabla^{\prime}\right)^{2}\left[\varepsilon^{+}+\left(\varepsilon^{+}\right)^{*}\right],
$$

where $*$ denotes the complex conjugate and $\varepsilon^{+}$ $=i \int_{0}^{+\infty} \widetilde{F}_{k}\left(\mathbf{r}^{\prime}, \mathbf{r}\right) k d k$ with

$$
\widetilde{F}_{k}\left(\mathbf{r}^{\prime}, \mathbf{r}\right)=\int_{V_{0}} d V_{0} \widetilde{G}_{k}^{(\text {out })}\left(\mathbf{r}^{\prime}, \mathbf{r}_{0}\right) \widetilde{G}_{k}^{(\text {in) }}\left(\mathbf{r}, \mathbf{r}_{0}\right) .
$$

In the planar geometry, we have $[1](\Delta z>0)$

$$
\begin{aligned}
\widetilde{G}_{k}^{(\mathrm{in})}\left(\mathbf{r}, \mathbf{r}_{0}\right)= & \frac{1}{(2 \pi)^{3}} \iint_{-\infty}^{+\infty} d u d v \exp (-i u \Delta x-i v \Delta y) \\
& \times\left\{-\chi\left(\frac{\rho}{2 k}\right) i \pi \operatorname{sgn}(k) \frac{\exp [-i \Delta z \operatorname{sgn}(k) w]}{w}\right. \\
& \left.-\chi\left(\frac{2 k}{\rho}\right) \pi \frac{\exp (-\Delta z w)}{w}\right\},
\end{aligned}
$$

and $\widetilde{G}_{k}^{\text {(out) }}\left(\mathbf{r}^{\prime}, \mathbf{r}_{0}\right)=\left[\widetilde{G}_{k}^{\text {(in) }}\left(\mathbf{r}^{\prime}, \mathbf{r}_{0}\right)\right]^{*}$ with the replacements of $\mathbf{r}$ $=(x, y, z)$ by $\mathbf{r}^{\prime}=\left(x^{\prime}, y^{\prime}, z^{\prime}\right), \rho$ by $\rho^{\prime}$, and $(u, v, w)$ by $\left(u^{\prime}, v^{\prime}, w^{\prime}\right)$, where $\Delta x=x-x_{0}$, etc. $\chi(\xi)=1$ for $|\xi|<1 / 2$ and 0 otherwise, $\operatorname{sgn}(k)=1$ for $k>0$ and -1 for $k<0, \rho=\sqrt{u^{2}+v^{2}}$, and $w=\sqrt{\left|k^{2}-\rho^{2}\right|}$. Here, we assume that the source is above the measurement plane $z_{0}=0$. Since $d V_{0}=d x_{0} d y_{0} d z_{0}$ and the identity $\iint_{-\infty}^{+\infty} d x_{0} d y_{0} \exp \left[i x_{0}\left(u-u^{\prime}\right)\right] \exp \left[i y_{0}\left(v-v^{\prime}\right)\right]$ $=(2 \pi)^{2} \delta\left(u-u^{\prime}\right) \delta\left(v-v^{\prime}\right)$, substituting $\widetilde{G}_{k}^{\text {(in) }}$ and $\widetilde{G}_{k}^{\text {(out) }}$ with the expansion of Eq. (14) into Eq. (11) gives $P^{(2)}\left(\mathbf{r}^{\prime}, \mathbf{r}\right)=0$.

In the spherical geometry, we have $(k>0)[10]$

$$
\widetilde{G}_{k}^{(\mathrm{in})}\left(\mathbf{r}, \mathbf{r}_{0}\right)=\frac{-i k}{4 \pi} \sum_{l=0}^{\infty}(2 l+1) j_{l}(k r) h_{l}^{(2)}\left(k r_{0}\right) P_{l}\left(\mathbf{n} \cdot \mathbf{n}_{0}\right)
$$

and $\widetilde{G}_{k}^{(\text {out })}\left(\mathbf{r}^{\prime}, \mathbf{r}_{0}\right)=\left[\widetilde{G}_{k}^{(\text {in) }}\left(\mathbf{r}^{\prime}, \mathbf{r}_{0}\right)\right]^{*}$ with the replacement of $\mathbf{n}$ by $\mathbf{n}^{\prime}$, where $j_{l}(\cdot)$ is a spherical Bessel function of the first kind, $h_{l}^{(2)}(\cdot)$ is a spherical Hankel function of the second kind, $P_{l}(\cdot)$ is a Legendre polynomial, and $\mathbf{n}^{\prime}=\mathbf{r}^{\prime} / r^{\prime}, \mathbf{n}_{0}$ $=\mathbf{r}_{0} / r_{0}$, and $\mathbf{n}=\mathbf{r} / r$. Since $d V_{0}=r_{0}^{2} d r_{0} d \Omega_{0}$ and the identity [10] $\quad \int_{\Omega_{0}} d \Omega_{0} P_{l}\left(\mathbf{n} \cdot \mathbf{n}_{0}\right) P_{l^{\prime}}\left(\mathbf{n}^{\prime} \cdot \mathbf{n}_{0}\right)=4 \pi \delta_{l l^{\prime}} P_{l}\left(\mathbf{n} \cdot \mathbf{n}^{\prime}\right) /(2 l+1)$, substituting $\widetilde{G}_{k}^{(\text {in) }}$ and $\widetilde{G}_{k}^{\text {(out) }}$ with the expansion of Eq. (15) into Eq. (13) gives

$$
\begin{aligned}
\widetilde{F}_{k}\left(\mathbf{r}^{\prime}, \mathbf{r}\right)= & \frac{k^{2}}{4 \pi} \sum_{l=0}^{\infty}(2 l+1) j_{l}(k r) j_{l}\left(k r^{\prime}\right) P_{l}\left(\mathbf{n} \cdot \mathbf{n}^{\prime}\right) \\
& \times \int_{r_{0}} r_{0}^{2} d r_{0} m_{l}^{2}\left(k r_{0}\right),
\end{aligned}
$$

with $m_{l}^{2}\left(k r_{0}\right)=j_{l}^{2}\left(k r_{0}\right)+n_{l}^{2}\left(k r_{0}\right)$, where $n_{l}(\cdot)$ is a spherical Bessel function of the second kind. Thus, $\widetilde{F}_{k}\left(\mathbf{r}^{\prime}, \mathbf{r}\right)$ is real and $\varepsilon^{+}(\mathbf{r})$ becomes purely imaginary. Therefore, from Eq. (12), $P^{(2)}\left(\mathbf{r}^{\prime}, \mathbf{r}\right)=0$.

In the cylindrical geometry, we denote $\mathbf{r}^{\prime}=\left(\rho^{\prime}, \varphi^{\prime}, z^{\prime}\right), \mathbf{r}$ $=(\rho, \varphi, z)$, and $\mathbf{r}_{0}=\left(\rho_{0}, \varphi_{0}, z_{0}\right)$. In this case, we have $(k>0)$ $[1,11]$

$$
\begin{aligned}
\widetilde{G}_{k}^{(\mathrm{in})}\left(\mathbf{r}, \mathbf{r}_{0}\right)= & \frac{1}{4 \pi^{2}} \sum_{n=-\infty}^{+\infty} \exp \left[i n\left(\varphi_{0}-\varphi\right)\right] \int_{-\infty}^{+\infty} d k_{z} \exp \left[i k_{z}\left(z_{0}-z\right)\right] \\
& \times\left[\frac{-i \pi}{2} \chi\left(\frac{k_{z}}{2 k}\right) J_{n}(\mu \rho) H_{n}^{(2)}\left(\mu \rho_{0}\right)\right. \\
& \left.+\chi\left(\frac{2 k}{k_{z}}\right) I_{n}(\mu \rho) K_{n}\left(\mu \rho_{0}\right)\right]
\end{aligned}
$$

and $\widetilde{G}_{k}^{\text {(out) }}\left(\mathbf{r}^{\prime}, \mathbf{r}_{0}\right)=\left[\widetilde{G}_{k}^{\text {(in) }}\left(\mathbf{r}^{\prime}, \mathbf{r}_{0}\right)\right]^{*}$ with the replacements of $n$ by $n^{\prime}, k_{z}$ by $k_{z}^{\prime}$, and $\mu$ by $\mu^{\prime}$, respectively, where $\mu$ $=\sqrt{\left|k^{2}-k_{z}^{2}\right|}$ and $\mu^{\prime}=\sqrt{\left|k^{2}-k_{z}^{\prime 2}\right|}, J_{n}(\cdot)$ is a Bessel function of the first kind, $H_{n}^{(2)}(\cdot)$ is a Hankel function of the second kind, $I_{n}(\cdot)$ is a modified Bessel function of the first kind, and $K_{n}(\cdot)$ is a modified Bessel function of the second kind. Since the identity $\quad[10] \quad \int_{\varphi_{0}} d \varphi_{0} \exp \left[i \varphi_{0}\left(n-n^{\prime}\right)\right] \int_{z_{0}} d z_{0} \exp \left[i z_{0}\left(k_{z}-k_{z}^{\prime}\right)\right]$ $=(2 \pi)^{2} \delta_{n n^{\prime}} \delta\left(k_{z}-k_{z}^{\prime}\right)$ and $d V_{0}=\rho_{0} d \rho_{0} d \varphi_{0} d z_{0}$, substituting $\widetilde{G}_{k}^{\text {(in) }}$ and $\widetilde{G}_{k}^{\text {(out) }}$ with the expansion of Eq. (17) into Eq. (13) gives

$$
\begin{aligned}
\tilde{F}_{k}\left(\mathbf{r}^{\prime}, \mathbf{r}\right)= & \frac{1}{4 \pi^{2}} \sum_{n=-\infty}^{+\infty} \exp \left[i n\left(\varphi^{\prime}-\varphi\right)\right] \int_{-\infty}^{+\infty} d k_{z} \exp \left[i k_{z}\left(z^{\prime}-z\right)\right] \\
& \times\left[\frac{\pi^{2}}{4} \chi\left(\frac{k_{z}}{2 k}\right) J_{n}\left(\mu \rho^{\prime}\right) J_{n}(\mu \rho) \int_{\rho_{0}} d \rho_{0} M_{n}^{2}\left(\mu \rho_{0}\right)\right. \\
& \left.+\chi\left(\frac{2 k}{k_{z}}\right) I_{n}\left(\mu \rho^{\prime}\right) I_{n}(\mu \rho) \int_{\rho_{0}} d \rho_{0} K_{n}^{2}\left(\mu \rho_{0}\right)\right]
\end{aligned}
$$

with $M_{n}^{2}\left(\mu \rho_{0}\right)=J_{n}^{2}\left(\mu \rho_{0}\right)+N_{n}^{2}\left(\mu \rho_{0}\right)$, where $N_{n}(\cdot)$ is a Bessel function of the second kind. It is easy to show that $\widetilde{F}_{k}\left(\mathbf{r}^{\prime}, \mathbf{r}\right)$ is real. Thus, $\varepsilon^{+}(\mathbf{r})$ is purely imaginary. From Eq. (12), $P^{(2)}\left(\mathbf{r}^{\prime}, \mathbf{r}\right)=0$.

In conclusion, we get $\mathrm{P}\left(\mathbf{r}^{\prime}, \mathbf{r}\right)=\delta\left(\mathbf{r}^{\prime}-\mathbf{r}\right)$ for all three common geometries. Therefore, from Eq. (4), we prove $p_{0}^{(b)}(\mathbf{r})$ $=p_{0}(\mathbf{r})$. Particularly for the planar geometry, by taking the limit $S_{0}^{\prime} \rightarrow \infty$ in Eq. (3), we find that the integral over $S_{0}^{\prime}$ gives 


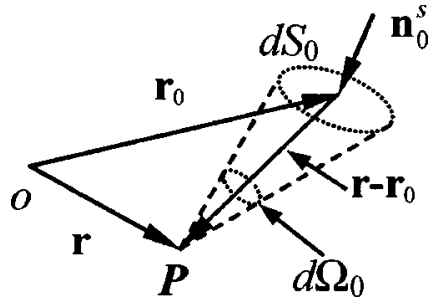

FIG. 2. Diagram of the solid angle $d \Omega_{0}$ for a detection element $d S_{0}$ with respect to a point $P$ at $\mathbf{r}$.

$p_{0}(\mathbf{r}) / 2$. Since the relationship $\boldsymbol{\nabla}_{0} \widetilde{G}_{k}^{(\mathrm{in})}\left(\mathbf{r}, \mathbf{r}_{0}\right)=-\boldsymbol{\nabla}$ $\times \widetilde{G}_{k}^{(\text {in) }}\left(\mathbf{r}, \mathbf{r}_{0}\right)$, by taking the inverse Fourier transform of $\tilde{p}\left(\mathbf{r}_{0}, k\right)$, we rewrite Eq. (3) in the time domain as

$$
p_{0}^{(b)}(\mathbf{r})=-\frac{2}{\Omega_{0}} \boldsymbol{\nabla} \cdot \int_{S_{0}} \mathbf{n}_{0}^{s} d S_{0}\left[\frac{p\left(\mathbf{r}_{0}, \bar{t}\right)}{\bar{t}}\right]_{\bar{t}=\left|\mathbf{r}-\mathbf{r}_{0}\right|},
$$

where $\Omega_{0}$ is a solid angle of the whole surface $S_{0}$ with respect to the reconstruction point inside $S_{0}: \Omega_{0}=2 \pi$ for the planar geometry and $\Omega_{0}=4 \pi$ for the spherical and cylindrical geometries. In addition, as mentioned before, a similar inversion formula for the spherical geometry was reported in Ref. [7]. We find that it can be simplified to Eq. (19) [14].

Further, we can rewrite Eq. (19) in a clear back-projection form as

$$
p_{0}^{(b)}(\mathbf{r})=\int_{\Omega_{0}} b\left(\mathbf{r}_{0}, \bar{t}=\left|\mathbf{r}-\mathbf{r}_{0}\right|\right) d \Omega_{0} / \Omega_{0},
$$

where $\quad b\left(\mathbf{r}_{0}, \bar{t}\right)=2 p\left(\mathbf{r}_{0}, \bar{t}\right)-2 \bar{t} \partial p\left(\mathbf{r}_{0}, \bar{t}\right) / \partial \bar{t} \quad$ is the backprojection term related to the measurement at position $\mathbf{r}_{0}$ and $d \Omega_{0}=d S_{0} /\left|\mathbf{r}-\mathbf{r}_{0}\right|^{2} \cdot\left[\mathbf{n}_{0}^{s} \cdot\left(\mathbf{r}-\mathbf{r}_{0}\right) /\left|\mathbf{r}-\mathbf{r}_{0}\right|\right]$ is the solid angle for a detection element $d S_{0}$ with respect to a reconstruction point $P$ at $\mathbf{r}$ (Fig. 2). The term $d \Omega_{0} / \Omega_{0}$ is a factor weighting the contribution to the reconstruction from the detection element $d S_{0}$. The reconstruction simply projects the quantity $b\left(\mathbf{r}_{0}, \vec{t}\right)$ backward on a spherical surface with respect to position $\mathbf{r}_{0}$. The first derivative over time $t$ actually represents a pure ramp filter $k$ in the frequency domain. The ramp filter depresses the low-frequency signal. It is not surprising that the relatively high-frequency components of the PA signal play the primary role in the reconstruction of the acoustic source inside the tissue. In the special case when $k\left|\mathbf{r}-\mathbf{r}_{0}\right| \gg 1$, $\bar{t} \partial p\left(\mathbf{r}_{0}, \bar{t}\right) / \partial \bar{t} \gg p\left(\mathbf{r}_{0}, \bar{t}\right)$; therefore, $b\left(\mathbf{r}_{0}, \bar{t}\right) \approx-2 \bar{t} \partial p\left(\mathbf{r}_{0}, \bar{t}\right) / \partial \bar{t}$.

\section{IMPLEMENTATION OF THE ALGORITHM}

Next, we discuss how to implement the BP algorithm. Usually, the EM pulse $I_{e}(t)$ is not a $\delta$ function. Thus, the right side of Eq. (1) should be replaced by $-p_{0}(\mathbf{r}) d I_{e}(t) / d t$, since the thermal diffusion effect is negligible in most soft tissues [15]. Consider a detector with an impulse response of $I_{d}(t)$; the measurement can be written as $p_{d}^{\prime}\left(\mathbf{r}_{0}, t\right)=H(t)$ $\otimes p\left(\mathbf{r}_{0}, t\right)$, where $H(t)=I_{e}(t) \otimes I_{d}(t)$ and $\otimes$ denotes a convolution. Thus, the spectrum of the measurement can be expressed by $\tilde{p}_{d}^{\prime}\left(\mathbf{r}_{0}, k\right)=\tilde{H}(k) \tilde{p}\left(\mathbf{r}_{0}, k\right)$, where $\tilde{H}(k)$ is the Fourier transform of $H(\bar{t})$. Since $H(\bar{t})$ is real, $\tilde{H}(-k)=[\tilde{H}(k)]^{*}$. Replacing $\tilde{p}\left(\mathbf{r}_{0}, k\right)$ with $\tilde{p}_{d}^{\prime}\left(\mathbf{r}_{0}, k\right)$ in Eq. (3) introduces the factor $\widetilde{H}(k)$ in the PSF expressed by Eq. (5), or both in the first term of the PSF by Eq. (10) and in the second term by Eq. (11). If $\tilde{H}(k)$ is even $[\tilde{H}(-k)=\tilde{H}(k)]$, it is easy to show $P^{(2)}\left(\mathbf{r}^{\prime}, \mathbf{r}\right)$ $=0$. Thus, the PSF equals $P^{(1)}\left(\mathbf{r}^{\prime}, \mathbf{r}\right)=-(1 / 2 \pi R) d H(R) / d R$ (with $R=\left|\mathbf{r}-\mathbf{r}^{\prime}\right|$ ), which is identical to the result in Ref. [1]. If $\tilde{H}(k)$ is odd $[\tilde{H}(-k)=-\tilde{H}(k)], P^{(1)}\left(\mathbf{r}^{\prime}, \mathbf{r}\right)=0$. Usually, however, $P^{(2)}\left(\mathbf{r}^{\prime}, \mathbf{r}\right) \neq 0$. In this case, the BP formula, Eq. (19), gives a "bad" reconstruction, because $P^{(2)}\left(\mathbf{r}^{\prime}, \mathbf{r}\right)$ does not converge to a point as $P^{(1)}\left(\mathbf{r}^{\prime}, \mathbf{r}\right)$ does. In other words, because acoustic pressure is phase sensitive, the reconstruction may be seriously destroyed due to the phase distortions that are introduced in the measured PA signals by $\tilde{H}(k)$. Moreover, the ramp filter $k$ clearly indicates the contribution of each frequency component in the reconstruction. If the $k$ weighting in the different frequency components is not followed, the reconstruction will also be distorted.

Therefore, to accurately recover the source distribution, in principle we need to find a filter to adjust the measurement. Two types of filters are possible. One is to restore the pressure by $\widetilde{F}_{1}(k)$ such that $\widetilde{F}_{1}(k) \tilde{H}(k)=1$ and $\widetilde{F}_{1}(k) \tilde{p}_{d}^{\prime}\left(\mathbf{r}_{0}, k\right)$ $=\tilde{p}\left(\mathbf{r}_{0}, k\right)$, and the other is to restore the derivative of the pressure by $\tilde{F}_{2}(k)$ such that $\tilde{F}_{2}(k) \tilde{H}(k)=-i k$ and $\tilde{F}_{2}(k) \tilde{p}_{d}^{\prime}\left(\mathbf{r}_{0}, k\right)=-i k \tilde{p}\left(\mathbf{r}_{0}, k\right)$. Since the real measurement is band limited, we need to add a low-pass filter, such as a Hanning window, to dampen the noisy high-frequency components. Sometimes, we also need to remove a small portion of the low-frequency components if the ultrasound detectors are not sensitive in that frequency range. For convenience, we denote the additional bandpass filter as $\tilde{W}(k)$. With the filter $\widetilde{F}_{1}(k)$, we compute $S_{1}^{(1)}\left(\mathbf{r}_{0}, \bar{t}\right)=F^{-1}\left[\tilde{W}(k) \widetilde{F}_{1}(k) \tilde{p}_{d}^{\prime}\left(\mathbf{r}_{0}, k\right)\right]$ and $S_{1}^{(2)}\left(\mathbf{r}_{0}, \vec{t}\right)=F^{-1}\left[-i k \widetilde{W}(k) \tilde{F}_{1}(k) \tilde{p}_{d}^{\prime}\left(\mathbf{r}_{0}, k\right)\right]$, where the Fourier transform $F^{-1}[(\cdot)]=(1 / 2 \pi) \int_{-\infty}^{+\infty}(\cdot) \exp (-i k \vec{t}) d k$ can be performed by the fast Fourier transform algorithm. Thus, the back-projection term is $b\left(\mathbf{r}_{0}, \bar{t}\right)=2 S_{1}^{(1)}\left(\mathbf{r}_{0}, \bar{t}\right)-2 \bar{t} S_{1}^{(2)}\left(\mathbf{r}_{0}, \bar{t}\right)$. With the filter $\tilde{F}_{2}(k)$, we first compute $S_{2}^{(2)}\left(\mathbf{r}_{0}, \vec{t}\right)$ $=F^{-1}\left[\tilde{W}(k) \widetilde{F}_{2}(k) \tilde{p}_{d}^{\prime}\left(\mathbf{r}_{0}, k\right)\right]$ and then $S_{2}^{(1)}\left(\mathbf{r}_{0}, \bar{t}\right)=\int_{0}^{\bar{t}} S_{2}^{(2)}\left(\mathbf{r}_{0}, \bar{t}\right) d \bar{t}$. Thus, the back-projection term is $b\left(\mathbf{r}_{0}, \vec{t}\right)=2 S_{2}^{(1)}\left(\mathbf{r}_{0}, \vec{t}\right)$ $-2 \bar{t} S_{2}^{(2)}\left(\mathbf{r}_{0}, \bar{t}\right)$. In addition, instead of the above frequencydomain filters, we can directly construct the corresponding time-domain filters.

In practical measurement, only a limited space around the tissue sample is available for ultrasound detection. For example, it is only possible to use a half-spherical measurement surface to image a human breast, in which the solid angle for all detectors on the half spherical surface with respect to a location inside the breast is often less than $4 \pi$ and may also vary at different locations. Thus, for sources at different locations but with the same intensities, the amplitudes in the reconstruction image will vary at different locations as well, which will cause a reconstruction distortion. A straightforward way to compensate for this kind of reconstruction distortion resulting from the limited view is to normalize the reconstruction at each location by a total solid angle as shown in Eq. (20). 


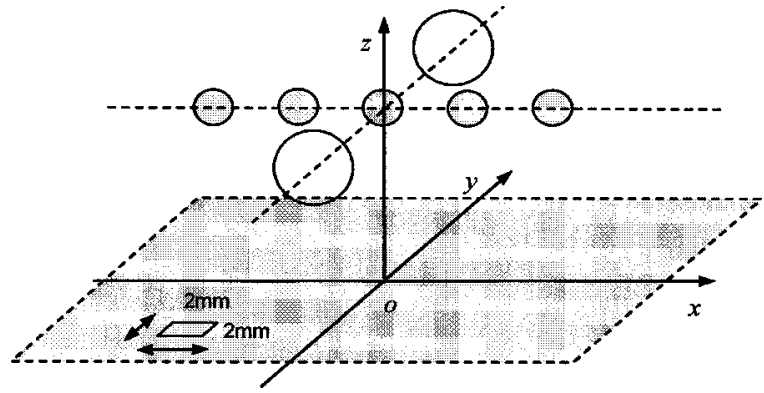

FIG. 3. Diagram of planar measurement geometry. See the text for detail.

Suppose there are $N$ detection positions with smallaperture detectors in which the $i$ th measurement is located at position $\mathbf{d}_{i}$ on the measurement surface $\mathbf{r}_{0}$ and occupies an area $\Delta S_{i}(i=1, \ldots, N)$ and $p_{d}^{\prime}\left(\mathbf{d}_{i}, \vec{t}\right)$ is the corresponding pressure measured by the $i$ th detector. Then, the back-projection formula, Eq. (20), can be rewritten in a discrete form as

$$
p_{0}^{(b)}(\mathbf{r})=\sum_{i=1}^{N} \Delta \Omega_{i} \times b\left(\mathbf{d}_{i}, \bar{t}=\left|\mathbf{d}_{i}-\mathbf{r}\right|\right) / \sum_{i=1}^{N} \Delta \Omega_{i},
$$

where $b\left(\mathbf{d}_{i}, \bar{t}\right)$ is computed from $p_{d}^{\prime}\left(\mathbf{d}_{i}, \bar{t}\right)$ by the method as discussed above and

$$
\Delta \Omega_{i}=\frac{\Delta S_{i}}{\left|\mathbf{r}-\mathbf{d}_{i}\right|^{2}} \cdot\left[\mathbf{n}_{0 i}^{s} \cdot \frac{\left(\mathbf{r}-\mathbf{d}_{i}\right)}{\left|\mathbf{r}-\mathbf{d}_{i}\right|}\right],
$$

in which $\mathbf{n}_{0 i}^{s}$ is the normal of the measurement surface at position $\mathbf{d}_{i}$ and points to the photoacoustic source distribution.

\section{NUMERICAL SIMULATION}

Next, we want to use numerical simulation to test the proposed algorithm. For simplicity, we consider uniform spherical absorbers surrounded by a nonabsorbing background medium. Let $a$ and $\mathbf{r}_{a}$ denote the radius and center of a spherical absorber, respectively. The excited pressure distribution caused by a $\delta$ EM illumination in the absorber can be expressed by $p_{0}(\mathbf{r})=A_{0} U\left(a-\left|\mathbf{r}-\mathbf{r}_{a}\right|\right)$, where $A_{0}$ is the intensity and $U(x)=1$ when $x>0$ and $U(x)=0$ when $x<0$. Then, the photoacoustic signal irradiated from this uniform sphere can be calculated by $p\left(\mathbf{r}_{0}, t\right)=A_{0} U(a-|R-c t|)(R$ $-c t) /(2 R)$, where $R$ is the distance between the detection position $\mathbf{r}_{0}$ and the absorber center $\mathbf{r}_{a}\left(R=\left|\mathbf{r}_{0}-\mathbf{r}_{a}\right|\right)$ [9]. For convenience, we use the center of the absorber to denote its position.

The numerical phantom sample is shown in Fig. 3. Five absorbers are evenly distributed at a distance of $15 \mathrm{~mm}$ along the horizontal line $(y=0$ and $z=15 \mathrm{~mm})$ that is parallel to the $x$ axis, and each absorber has a radius of $1.5 \mathrm{~mm}$ and an intensity of 1 . Another two absorbers are located at a distance of $15 \mathrm{~mm}$ along a horizontal line $(x=0$ and $z$ $=15 \mathrm{~mm}$ ) that is parallel to the $y$ axis, and each absorber has a radius of $4 \mathrm{~mm}$ and an intensity of 1. [See the detailed positions of these absorbers in the text below Eq. (23).]
Without loss of generality, we can take the planar measurement surface as an example. As shown in Fig. 3, a rectangular-shaped detector $2 \mathrm{~mm} \times 2 \mathrm{~mm}$, scans the samples in the $z=0$ plane along both the $x$ and $y$ axes from $-30 \mathrm{~mm}$ to $30 \mathrm{~mm}$ with a spatial sampling period of $2 / 3 \mathrm{~mm}$, where the center of the detector surface represents its position. In the computation of the PA signal of the detector, for simplicity, we evenly divide the detector surface $(2 \mathrm{~mm} \times 2 \mathrm{~mm})$ into 25 small elements. Then, the total signal received by the detector at $\mathbf{d}_{i}=\left(x_{d i}, y_{d i}, 0\right)$ can be computed by a summation as

$$
\begin{aligned}
p_{d}\left(\mathbf{d}_{i}, t\right)= & \sum_{m=-2}^{2} \sum_{n=-2}^{2} \frac{1}{25}\left[\sum_{j=1}^{5} \frac{U\left(1.5-\left|R_{i j}-c t\right|\right)\left(R_{i j}-c t\right)}{2 R_{i j}}\right. \\
& \left.+\sum_{j=6}^{7} \frac{U\left(4-\left|R_{i j}-c t\right|\right)\left(R_{i j}-c t\right)}{2 R_{i j}}\right],
\end{aligned}
$$

where $R_{i j}=\left|\mathbf{d}_{i}^{(m, n)}-\mathbf{r}_{a j}\right|, \mathbf{d}_{i}^{(m, n)}=\left(x_{d i}+0.4 m, y_{d i}+0.4 n, 0\right)$, and the center position of the $j$ th absorber $\mathbf{r}_{a j}(j=1, \ldots, 7)$ $=\left(x_{a j}, y_{a j}, z_{a j}\right): \quad( \pm 18,0,15),( \pm 9,0,15),(0,0,15), \quad$ and $(0, \pm 12,15) \mathrm{mm}$, respectively. In practice, the detection system is band limited. It is necessary to introduce a low-pass filter $\tilde{W}_{c}(k)$ characterized by a cutoff angular frequency $f_{c}$. A Hanning window is our choice in this case $(k=2 \pi f / c)$ :

$$
\tilde{W}_{c}(k)= \begin{cases}0.5+0.5 \cos \left(\pi \frac{f}{f_{c}}\right), & \text { if }|f|<f_{c}, \\ 0, & \text { otherwise. }\end{cases}
$$

Here, we choose $f_{c}=4 \mathrm{MHz}$ and the data sampling frequency as $20 \mathrm{MHz}$.

In the simulation, we first use Eq. (23) to compute $p_{d}\left(\mathbf{d}_{i}, \vec{t}\right)$ without additional noise. Then, we calculate $b\left(\mathbf{d}_{i}, \bar{t}\right)=2 F^{-1}\left[\tilde{W}_{c}(k) \tilde{p}_{d}\left(\mathbf{d}_{i}, k\right)\right]-2 \bar{t} F^{-1}\left[-i k \tilde{W}_{c}(k) \tilde{p}_{d}\left(\mathbf{d}_{i}, k\right)\right]$ and further use Eq. (21) to compute the reconstruction. Figure $4(\mathrm{a})$ is a gray scale image of the reconstructed distribution in the $z=15 \mathrm{~mm}$ plane, and Figs. 4(b) and 4(c) show a comparison between the original and reconstructed distributions along the lines $y=0$ and $x=0 \mathrm{~mm}$, respectively. The reconstructed values are in good agreement with the original ones. Due to the limited-angle view and the limited bandwidth, some discrepancies occur in the reconstruction, but they are minimal.

We also compute the reconstruction from the data with additional noise: $p_{d}\left(\mathbf{d}_{i}, \bar{t}\right) \leftarrow p_{d}\left(\mathbf{d}_{i}, \bar{t}\right)+0.1 c_{\mathrm{RAND}}$, where $c_{\mathrm{RAND}}$ is a series of computer-generated random numbers in the range of $(-1,1)$. Since the initial pressure intensity of each absorber is 1, 0.1 is a significant noise level. Figure 5(a) is a gray scale image of the reconstructed distribution in the $z$ $=15 \mathrm{~mm}$ plane, and Figs. 5(b) and 5(c) show a comparison between the original and reconstructed distributions along the lines $y=0$ and $x=0 \mathrm{~mm}$, respectively. The reconstructed values are also in good agreement with the original ones. This example indicates that the proposed back-projection algorithm is very insensitive to the random noise. 


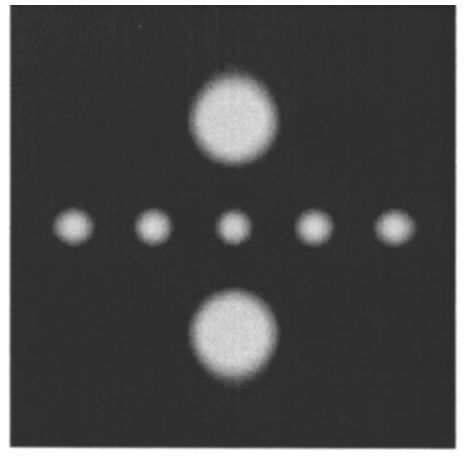

(a)

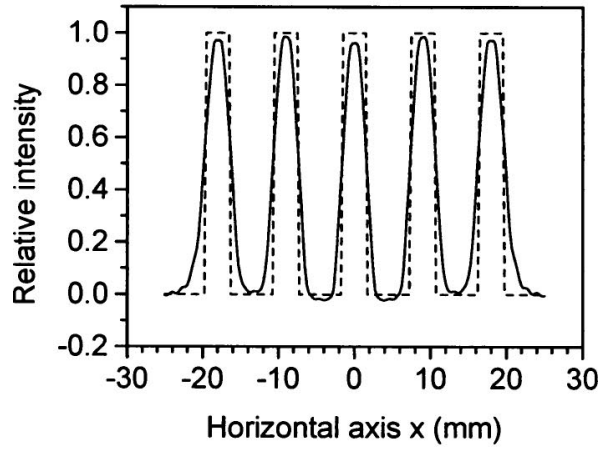

(b)

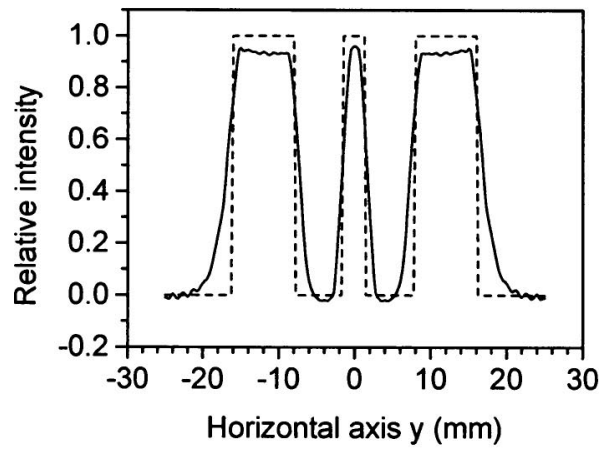

(c)

FIG. 4. Reconstruction without noise. (a) Gray scale image of the reconstructed pressure distribution in the $z=15 \mathrm{~mm}$ plane. Comparison between the original (dashed line) and reconstructed (solid line) distributions: (b) along $y=0 \mathrm{~mm}$ and (c) along $x=0 \mathrm{~mm}$.

\section{CONCLUSION}

We have presented in this paper a unified and exact timedomain back-projection algorithm for the three common measurement geometries with the assumption of constant sound speed. We can extend this algorithm straightforwardly to the limited-angle view case, in which the reconstruction may be incomplete and reconstruction artifacts may occur. The solid-angle weighting factor in the BP formula, however, can compensate for the variations in the detection views. It has to be pointed out that significant acoustic inhomogeneity of the sample may introduce reconstruction distortions, which, however, may be corrected or minimized using

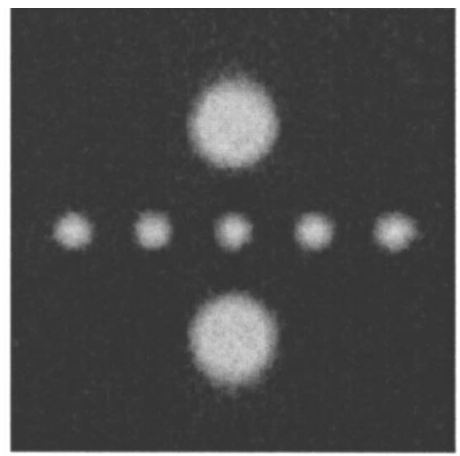

(a)

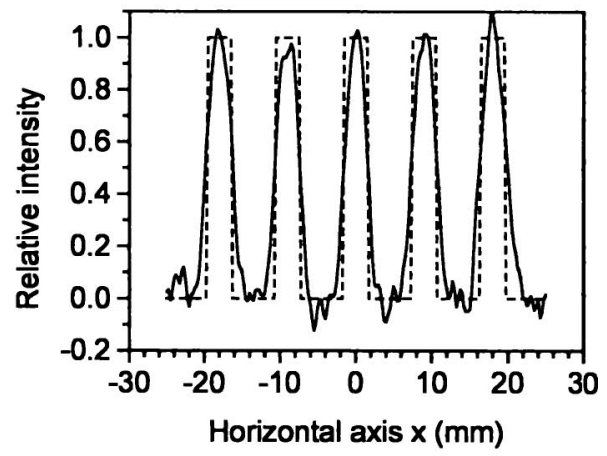

(b)

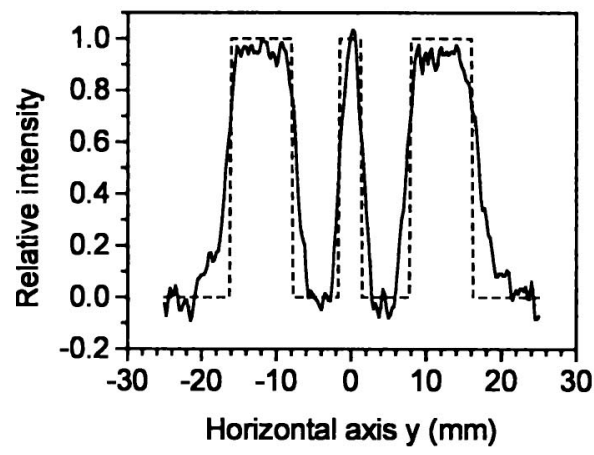

(c)

FIG. 5. Reconstruction with noise. (a) Gray scale image of the reconstructed pressure distribution in the $z=15 \mathrm{~mm}$ plane. Comparison between the original (dashed line) and reconstructed (solid line) distributions: (b) along $y=0 \mathrm{~mm}$ and (c) along $x=0 \mathrm{~mm}$.

modified algorithms that take account of the acoustic inhomogeneity.

This BP formula can serve as a basis for time-domain photoacoustic reconstruction in three-dimensional space. In principle, this algorithm can also be extended to other inverse-source diffraction tomographies.

\section{ACKNOWLEDGMENTS}

This project was sponsored in part by U.S. Army Medical Research and Materiel Command Grant No. DAMD17-00-10455, National Institutes of Health Grant Nos. R01 EB000712 and R01 NS46214, and Texas Higher Education Coordinating Board Grant No. ARP 000512-0063-2001. 
[1] M.-H. Xu and L.-H. V. Wang, Phys. Rev. E 67, 056605, (2003).

[2] R. A. Kruger, D. R. Reinecke, and G. A. Kruger, Med. Phys. 26, 1832 (1999).

[3] M.-H. Xu and L.-H. V. Wang, IEEE Trans. Med. Imaging 21, 814 (2002)

[4] M.-H. Xu, Y. Xu, and L.-H. V. Wang, IEEE Trans. Biomed. Eng. 50, 1086 (2003).

[5] A complete list of references is too extensive to reproduce here. See Refs. [3,4].

[6] A. C. Kak and M. Slaney, Principles of Computerized Tomographic Imaging (SIAM, Philadelphia, 2001).

[7] D. Finch, S. K. Patch, and Rakesh, SIAM J. Math. Anal. 35, 1213 (2003).

[8] V. E. Gusev and A. A. Karabutov, Laser Optoacoustics (AIP, New York, 1993).

[9] G. J. Diebold, T. Sun, and M. I. Khan, Phys. Rev. Lett. 67, 3384 (1991).

[10] G. B. Arfken and H. J. Weber, Mathematical Methods for Physicists (Academic, San Diego, 1995).

[11] P. M. Morse and K. U. Ingard, Theoretical Acoustics (McGraw-Hill, New York, 1968).

[12] J. D. Jackson, Classical Electrodynamics (Wiley, New York, 1975).

[13] In the planar geometry $\left(S_{0}: z_{1}=0\right.$ and $S_{0}^{\prime}$ at infinity), let $R_{ \pm}=\sqrt{\left(x-x_{1}\right)^{2}+\left(y-y_{1}\right)^{2}+\left(z \pm z_{1}\right)^{2}}, \quad \widetilde{G}_{k}^{(D)}\left(\mathbf{r}, \mathbf{r}_{1}\right)=(1 / 4 \pi)$ $\times\left[\exp \left(-i k R_{-}\right) / R_{-}-\exp \left(-i k R_{+}\right) / R_{+}\right]$(p. 430 in [12]). By taking the limit $S_{0}^{\prime} \rightarrow \infty$, the integral over surface $S_{0}^{\prime}$ gives $p_{0}(\mathbf{r}) / 2$. For the other two geometries, we construct $\widetilde{G}_{k}^{(D)}\left(\mathbf{r}, \mathbf{r}_{1}\right)$
$=\widetilde{G}_{k}^{(\text {in })}\left(\mathbf{r}, \mathbf{r}_{1}\right)+\widetilde{\psi}_{k}\left(\mathbf{r}, \mathbf{r}_{1}\right), \quad$ where $\quad \tilde{\psi}_{k}\left(\mathbf{r}, \mathbf{r}_{1}\right) \quad$ satisfies $\left(\boldsymbol{\nabla}^{2}+k^{2}\right) \widetilde{\psi}_{k}\left(\mathbf{r}, \mathbf{r}_{1}\right)=0 \quad$ (p. 320 in [11]). In the spherical geometry $\left(S_{0}: r_{1}=r_{0}\right)$, for $k>0, \widetilde{G}_{k}^{(D)}\left(\mathbf{r}, \mathbf{r}_{1}\right)=(-i k / 4 \pi) \Sigma_{l=0}^{\infty}(2 l$ $+1) j_{l}(k r) P_{l}\left(\mathbf{n} \cdot \mathbf{n}_{1}\right)\left[h_{l}^{(2)}\left(k r_{1}\right)-h_{l}^{(1)}\left(k r_{1}\right) h_{l}^{(2)}\left(k r_{0}\right) / h_{l}^{(1)}\left(k r_{0}\right)\right]$. In the cylindrical geometry $\left(S_{0}: \rho_{1}=\rho_{0}\right)$, denoting $\mu$ $=\sqrt{k^{2}-k_{z}^{2}}\left(k>k_{z}\right) \quad$ and $\quad \mu=i \sqrt{k_{z}^{2}-k^{2}}\left(k<k_{z}\right)$, for $k>0, \quad \widetilde{G}_{k}^{(D)}\left(\mathbf{r}, \mathbf{r}_{1}\right)=\left(1 / 4 \pi^{2}\right) \sum_{n=-\infty}^{\infty} \exp \left[\operatorname{in}\left(\varphi_{1}-\varphi\right)\right] \int_{-\infty}^{+\infty} d k_{z}$ $\exp \left[i k_{z}\left(z_{1}-z\right)\right] \widetilde{g}_{n}\left(\rho, \rho_{1}, \rho_{0} ; \mu\right), \quad$ where $\quad \tilde{g}_{n}\left(\rho, \rho_{1}, \rho_{0} ; \mu\right)$ $=(-i \pi / 2) J_{n}\left(\mu^{*} \rho\right)\left[H_{n}^{(2)}\left(\mu^{*} \rho_{1}\right)-H_{n}^{(1)}\left(\mu \rho_{1}\right) H_{n}^{(2)}\left(\mu^{*} \rho_{0}\right) / H_{n}^{(1)}\left(\mu \rho_{0}\right)\right]$. Wronskian relations should be used for computing the derivatives of Bessel or Hankel functions.

[14] We rewrite Eq. (2) as $\bar{t} \int_{0}^{\bar{t}} p\left(\mathbf{r}_{0}, \bar{t}\right) d \bar{t}=(1 / 4 \pi) \iiint_{V} d^{3} r p_{0}(\mathbf{r}) \delta(\mid \mathbf{r}$ $\left.-\mathbf{r}_{0} \mid-\bar{t}\right)$. If we denote $\Psi\left(\mathbf{r}_{0}, \bar{t}\right)=\bar{t} \int_{0}^{\bar{t}} p\left(\mathbf{r}_{0}, \bar{t}\right) d \bar{t}$, the reconstruction formula for the spherical geometry can be written as [7] $p_{0}^{\prime}(\mathbf{r})=-2 /\left(r_{0} \Omega_{0}\right) \nabla^{2} \int_{S_{0}} d S_{0} \Psi\left(\mathbf{r}_{0},\left|\mathbf{r}-\mathbf{r}_{0}\right|\right) /\left|\mathbf{r}-\mathbf{r}_{0}\right|$. Since $\boldsymbol{\nabla} \mid \mathbf{r}$ $-\mathbf{r}_{0}\left|=\left(\mathbf{r}-\mathbf{r}_{0}\right) /\right| \mathbf{r}-\mathbf{r}_{0} \mid$, the above formula reduces to $p_{0}^{\prime}(\mathbf{r})$ $=p_{0}^{(b)}(\mathbf{r})-2 /\left(r_{0} \Omega_{0}\right) \boldsymbol{\nabla} \cdot \mathbf{r} F(\mathbf{r}), \quad$ where $\quad F(\mathbf{r})=\int_{S_{0}} d S_{0} p\left(\mathbf{r}_{0}, \mid \mathbf{r}\right.$ $\left.-\mathbf{r}_{0} \mid\right) /\left|\mathbf{r}-\mathbf{r}_{0}\right|=2 \int_{V^{\prime}} d^{3} r^{\prime} p_{0}\left(\mathbf{r}^{\prime}\right) \int_{-\infty}^{+\infty}(-i k) d k \int_{S_{0}} d S_{0} \widetilde{G}_{k}^{(\text {out })}\left(\mathbf{r}^{\prime}, \mathbf{r}_{0}\right)$ $\times \widetilde{G}_{k}^{\text {(in) }}\left(\mathbf{r}, \mathbf{r}_{0}\right)$. Similarly to prove $P^{(2)}\left(\mathbf{r}^{\prime}, \mathbf{r}\right)=0$, we find $F(\mathbf{r})$ $=0$. Therefore, $p_{0}^{\prime}(\mathbf{r})=p_{0}^{(b)}(\mathbf{r})$.

[15] Upon the absorption of a pulse energy, the thermal diffusion during the pulse period $\tau_{p}$ can be estimated by a thermal diffusion length: $\delta_{T}=2 \sqrt{D_{T} \tau_{p}}$ [A. C. Tam, Rev. Mod. Phys. 58, 381 (1986)], where $D_{T}$ is the thermal diffusivity. For most soft tissues, $D_{T} \sim 1.4 \times 10^{-3} \mathrm{~cm}^{2} / \mathrm{s}$ [F. A. Duck, Physical Properties of Tissue (Academic, London, 1990)]. For a pulse of $\tau_{p}$ $=0.5 \mu \mathrm{s}, \delta_{T} \approx 0.5 \mu \mathrm{m}$, which is typically much less than the spatial resolution of most PA imaging systems. 